"This is a post-peer-review, pre-copyedit version of an article published in Marine Systems \& Ocean Technology. The final authenticated version is available online at: http://dx.doi.org/10.1007/s40868-019-00056-1". 


\title{
Slingshot Resonance for Ocean Wave Energy Conversion
}

\author{
Francisco J. Arias ${ }^{a *}$ and Salvador De Las Heras ${ }^{a}$ \\ a Department of Fluid Mechanics, University of Catalonia, \\ ESEIAAT C/ Colom 11, 08222 Barcelona, Spain
}

(Dated: April 15, 2019)

\begin{abstract}
The slingshot effect and its application to converting ocean wave energy is discussed. It is shown that, owing to the large inertia transported by ocean waves and their periodicity, the slingshot effect can result in the transmission of significant kinetic energy to a puck colliding elastically with a pusher plate driven by ocean wave motion. A simplified geometrical model is used to demonstrate that, despite the stochastic nature of the collisions (whereby collisions occur at random times in the wave cycle), head-on collisions occur more frequently, yielding a net average gain of energy. However, the most promising configuration for applying the slingshot effect to ocean-wave energy conversion is that which matches, through appropriate design, the travel time of the puck between collisions with the wave period. Then, only head-on collisions occur, resulting in a significant magnification of the puck kinetic energy. Further research will be required before this slingshot effect can be practically implemented for ocean-wave energy conversion.
\end{abstract}

Keywords. Ocean wave energy conversion; Slingshot effect, Resonant cavities, Electromagnetic wave converters

\section{INTRODUCTION}

Considerable research effort has been devoted to extracting energy from ocean waves over the last two centuries [1]. More than 1000 techniques have been patented in North America, Japan, and Europe [2] over the past century, which fall into three main categories: attenuators, in which a floating device operates parallel to the wave propagation direction (effectively "riding" the waves); point absorbers, in which a floating structure absorbs energy from all directions through its motion at or near the water surface; and terminators, in which a device extends perpendicular to the direction of wave propagation and captures or reflects the power of the wave [3],[4]. To maximise the extraction yield, designers tune the conversion system to resonate with incoming waves [5]-[7], as it is well established that, under such conditions, the absorbing system attains maximal amplitude and speed, thereby optimising the conversion yield [8],[9]. A seminal example of a resonant cavity used for converting ocean-wave energy was by Masuda (1971) [10], who designed the first commercial resonator as a navigation aid. A full-scale wave energy resonator system was subsequently developed jointly by Canada, Ireland, Japan, the United Kingdom, and the United States with the support of the International Energy Agency (IEA). Since then, several methods have sought to match the ocean-wave frequency with the natural frequency of the absorber system, either actively or passively. Forcing a system to resonate through active control generally requires powerful braking mechanisms to latch the point absorber at fixed positions [11]-[13],

*Corresponding author: Tel.: +93 7398 666; Electronic address: frarias@mf .upc.edu together with predictive models [14]. Passive methods involve shifting the frequency response of the system by increasing the inertia of its moving parts [15],[16]. Despite being less efficient, passive methods are designed to use control and braking systems that are more economical and robust than those of active methods.

The objective of this work was to explore a novel concept of ocean energy conversion based on the so-called slingshot effect that can give rise to a new type of resonance, hereafter referred to as the slingshot resonance.

\section{BASIC PRINCIPLE}

\section{A. Slingshot effect}

The slingshot effect (outlined in Fig. 1) results from the elastic interaction between two colliding objects of mass $m_{1}$ and $m_{2}$ and velocity $u_{1}$ and $u_{2}$ relative to some reference inertial frame. It is assumed that $m_{1} \gg m_{2}$ and $m_{1} u_{1} \gg m_{2} u_{2}$. In an elastic collision, the total momentum and kinetic energy are conserved. The mass difference therefore implies minimal change in the velocity of body 1, while body 2 recoils with a new velocity $\grave{u}_{2}$ given by

$$
\grave{u}_{2}=u_{2}+2 u_{1} \text {. }
$$

The slingshot effect has important technological applications, for example in space technology. In a manoeuvre known as gravity assist (also called gravitational slingshot), the motion of a planet is exploited to redirect the trajectory of an approaching spacecraft. Manually effecting this redirection through propulsion would be prohibitively expensive, if not impossible. 


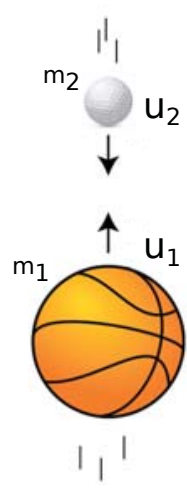

BEFORE

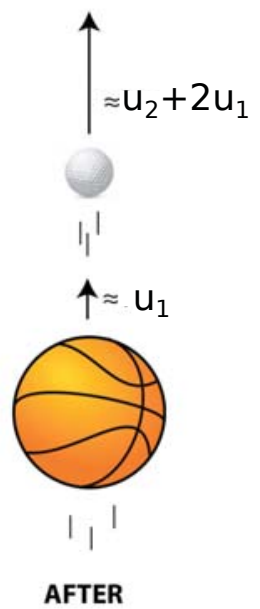

FIG. 1: Illustration of the slingshot effect.

\section{B. Slingshot effect for converting ocean wave energy}

Ocean waves carry considerable amounts of inertia. For instance, waves generated by a storm are capable of lifting the bow of a 200,000-tonne ship out of water. Furthermore, the periodic application of the slingshot effect at every wave cycle can result in a significant transmission of kinetic energy from the pusher plate and the puck in Fig. 2. This paper will outline a first assessment for this oceanic slingshot effect.

\section{METHOD}

Real ocean waves are generated mostly by local winds. The wave amplitude, frequency, and propagation direction therefore relate directly to the wind velocity and direction. However, the waves might appear to have a random amplitude, frequency, or direction [9].

Because of dispersion, long waves travel further than short waves away from the local stormy region that generated them. At large distances, the waves therefore appear as nearly monochromatic swells with long wavelengths. As a first approach, the present study therefore focuses on this regime for simplicity and also because energy conversion is most efficient when conducted under such monochromatic conditions.

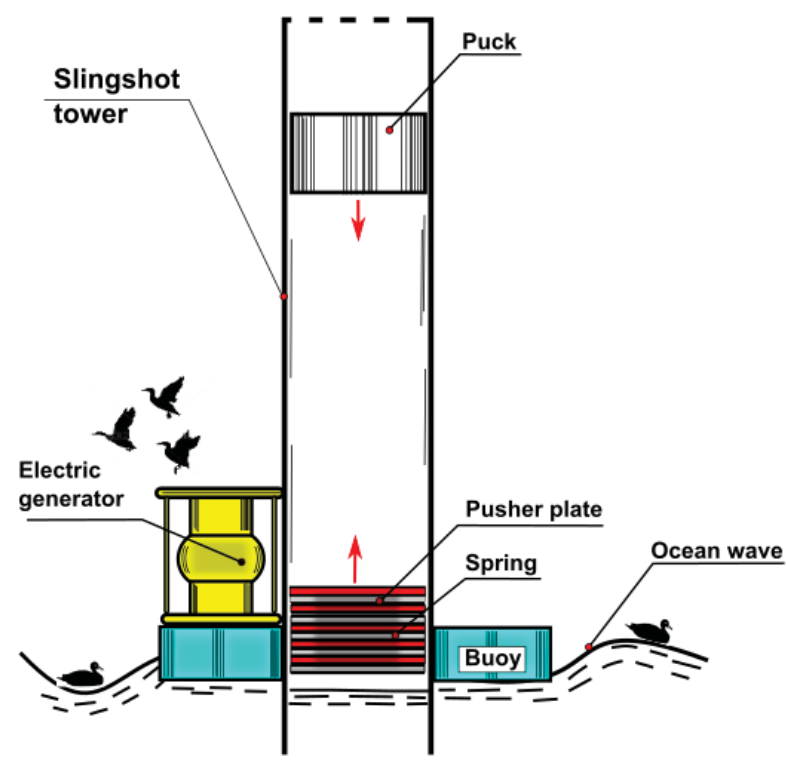

FIG. 2: Slingshot resonant tower.

\section{A. Stochastic mode}

The vertical velocity at the surface of an ocean wave is given as a function of time $t$ as [9]

$$
v=v_{o} \cos \omega t
$$

where $v_{o}$ is the velocity amplitude and $\omega$ the wave frequency. Thus, if the puck in Fig. 2 travels with velocity $u$ and collides with the pusher plate (which follows the wave surface), it will recoil with a final velocity $\grave{u}$ given by Eq.(1) as

$$
\grave{u}=u+2 v \text {. }
$$

Substituting Eq.(2) into Eq.(3), one obtains

$$
\grave{u}=u+2 v_{o} \cos \omega t .
$$

Because the stochastic process in which head-on and wave-away collisions take place, the mean square velocity is evaluated as the second-order moment of the velocity

$$
<\grave{u}^{2}>=\frac{1}{T} \int_{0}^{T}\left(u+2 v_{o} \cos \omega t\right)^{2} d t
$$


where $T$ is the period of the ocean wave.

\section{- Discussion I}

By assuming a constant puck velocity $u$ at collision, the integral in Eq.(5) yields

$$
<\grave{u}^{2}>=u^{2}+2 v_{o}^{2} .
$$

There is therefore a net gain of $\frac{E}{m}=v_{o}^{2}$ in the kinetic energy per unit mass of the puck ${ }^{m}$. This implies that, under conditions where collisions occur stochastically, head-on collisions occur more frequently on average.

Consider next the more realistic situation of a slingshot driven by ocean waves where the downward velocity of the puck is time-dependent. Let the puck be initially at rest at some height above the wave surface at time $t=0$, and fall freely under gravity to collide with the pusher plate (i.e., the wave) at time $t$ with a velocity $g t$, where $g$ is the acceleration due to gravity. The pusher plate at this moment has a velocity given by Eq.(2) as

$$
v=v_{o} \cos (\omega t+\delta),
$$

where $\delta \in[0,2 \pi]$ represents a phase shift. At the moment of collision, the puck recoils with a velocity that is given by

$$
\grave{u}=g t+2 v_{o} \cos (\omega t+\delta) .
$$

The second-order moment of the velocity

$$
<\grave{u}^{2}>=\frac{1}{2 \pi} \frac{1}{T} \int_{o}^{2 \pi} \int_{o}^{T}\left(g t+2 v_{o} \cos (\omega t+\delta)\right)^{2} d t d \delta
$$

is integrated to give

$$
<\grave{u}^{2}>=\frac{T^{2} g^{2}}{3}+2 v_{o}^{2} .
$$

The first term on the right side represents the energy needed to maintain the periodic operation of the system (i.e., continuously transforming kinetic into potential gravitational energy) and is not available as surplus of energy. The net energy per unit mass that can be extracted from the system at every collision is therefore given by $\Delta E=v_{o}^{2}$. This result is identical to that derived above.

\section{B. Slingshot resonance}

Consider now the more interesting case where the time between successive collisions is made to match the period of the wave exactly. The collisions occur at the same location and with the same velocities of the puck and pusher plate. Ideally, the pusher plate collides at its maximum velocity $v=v_{o}$.

This synchronisation between the puck and the wave can be realised by tuning several parameters, as discussed below. For the time being, let us assume that resonance conditions are satisfied with puck and pusher-plate velocities $u$ and $v_{o}$ at each head-on collision. From the previous discussions, the recoil puck velocity is given by

$$
\grave{u}=u+2 v_{o}
$$

and the second-order moment of the velocity becomes

$$
\begin{gathered}
<\grave{u}^{2}>=\frac{1}{T} \int_{o}^{T}\left(u+2 v_{o}\right)^{2} d t \quad \therefore \\
<\grave{u}^{2}>=u^{2}+4 u v_{o}+4 v_{o}^{2}
\end{gathered}
$$

The average extractable energy per collision and per unit mass of the puck is therefore given by

$$
\Delta E=2 u v_{o}+2 v_{o}^{2}
$$

\section{- Discussion II}

A clear difference between this result and the stochastic mode previously analysed (where the extractable energy per unit mass was $\Delta E=v_{o}^{2}$ ) is the additional term $2 u v_{o}$ that depends on the puck velocity $u$ at the moment of collision. Although at first sight it might seem that this velocity $u$ could be made to increase arbitrarily through successive collisions, this does not happen in reality. As discussed below, every increment in the kinetic energy of the puck must translate into a proportional increase in the maximum height within the cavity or tower (at least if the wave period is to equal the puck travel time). Eventually, the tower height would no longer be suitable. Moreover, as the kinetic energy of the puck becomes increasingly large, the assumption of the slingshot effect that the puck momentum should be small would become invalid. We will proceed to analyse the condition for the oceanic slingshot resonance in this perspective.

For illustration, consider first the minimal slingshot resonant system (tower or cavity) depicted in Fig. 3. After rising to its maximum height $h$, the puck starts to fall, colliding head-on after a time $t_{t}$ and at a velocity $u_{i}$ with the pusher plate traveling at its maximum velocity $v_{o}$. The puck then recoils upwards with velocity $u_{i}+2 v_{o}$.

We define the vertical length coordinate $z$ as being positive in the upward direction. The general equation 


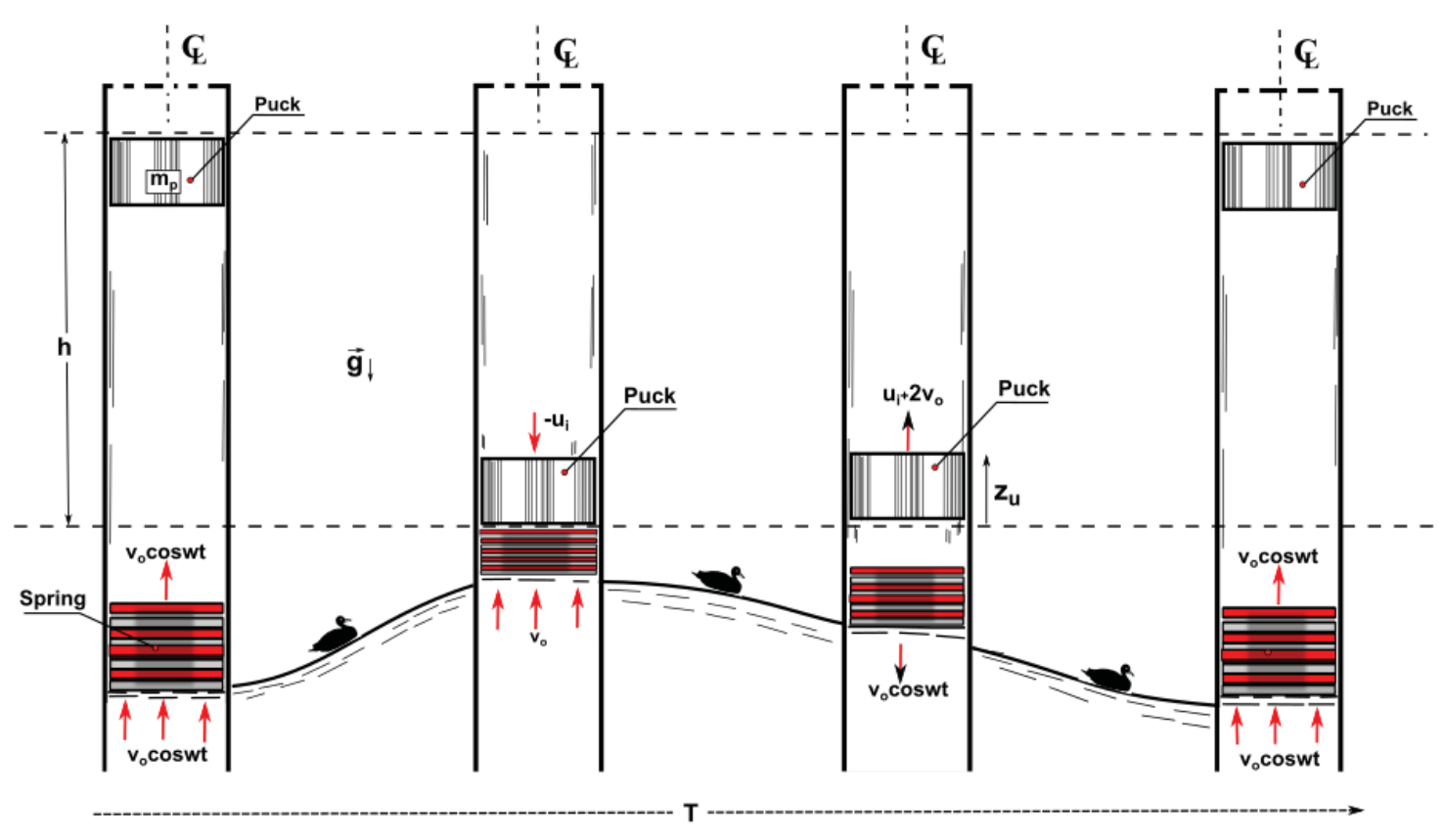

FIG. 3: Slingshot resonant tower

of motion for the puck, in the reference frame centred at the point of collision, is

$$
\dot{u}=-g-\frac{b u}{m_{p}}
$$

where $m_{p}$ is the puck mass, $\dot{u}$ and $u$ are its acceleration and velocity, respectively, and $b$ is a damping coefficient. Eq.(14) can then be rewritten as

$$
\dot{u}=-g-2 \beta u
$$

with $\beta$ defined by

$$
\beta=\frac{b}{2 m_{p}}
$$

For the purpose of the analysis, we amalgamate as "losses" all the contributions to the energy leaving the system during each cycle. These include the energy lost through friction and other irreversible processes, and the energy extracted for the conversion. This definition allows the definition of an effective damping parameter $\beta$ that covers both energy contributions:

$$
\beta=\beta_{f}+\beta_{c}
$$

where $\beta_{f}$ represents the damping due to irreversible losses (e.g., friction), and $\beta_{c}$ the effective damping associated with the converter (e.g., the turbine and electromagnetic inductor). This approximation is valid insofar as the resisting force experienced by the converter is proportional to the puck velocity, and provided that Eq.(16) can be used. As shown below, the latter condition is fulfilled for electromagnetic converters.

The solution of Eq.(15) is

$$
u=-\frac{g}{2 \beta}-C_{1} e^{-2 \beta t}
$$

where $C_{1}$ is a constant to be determined from initial conditions. Integrating Eq.(18), we have

$$
z=-\frac{g}{2 \beta} t+\frac{C_{1}}{2 \beta} e^{-2 \beta t}+C_{2}
$$

where $C_{2}$ is also a constant to be determined from initial conditions.

\section{Boundary and initial conditions}

The velocity $u$ can be found by considering the following boundary and initial conditions of the resonant system. If, at a certain position $z$ and time $t$, the puck is moving downwards with a velocity $-u_{i}$ and collides with the pusher plate moving upwards with a velocity $v_{o}$, the puck recoils upwards with a velocity $\approx u_{i}+2 v_{o}$, as previously discussed. If for convenience we define this position as $z=0$ and this time $t=0$, the initial and boundary conditions are specified as

$$
\begin{array}{ll}
\text { 1. } & z(t=0)=0 \\
\text { 2. } & u(t=0)=u_{i}+2 v_{o}
\end{array}
$$




$$
\begin{array}{ll}
\text { 3. } & z(t=T)=0 \\
\text { 4. } & u(t=T)=-u_{i}
\end{array}
$$

The two last conditions signify that, after a period $t=T$, the puck has returned to the same position as at the previous collision, i.e., $z=0$, and has a velocity $-u_{i}$, so that a further collision instantaneously changes its velocity to $u_{i}+2 v_{o}$ at $z=0$. The cycle can then be repeated indefinitely.

The first two conditions allow the determination of $C_{1}$ and $C_{2}$ in Eqs.(19) and (18), yielding

$$
\begin{gathered}
u=-\frac{g}{2 \beta}\left[1-e^{-2 \beta t}\right]+\left(u_{i}+2 v_{o}\right) e^{-2 \beta t} \\
z=-\frac{g}{2 \beta} t-\left[\frac{g}{4 \beta^{2}}+\frac{u_{i}+2 v_{o}}{2 \beta}\right] e^{-2 \beta t}+\frac{g}{4 \beta^{2}}+\frac{u_{i}+2 v_{o}}{2 \beta}
\end{gathered}
$$

while the third and fourth conditions provide two relationships between $\beta$ and $u_{i}$ :

$$
u_{i}=\left[\frac{g}{2 \beta}\left(1-e^{-2 \beta T}\right)-2 v_{o} e^{-2 \beta T}\right] \frac{1}{1+e^{-2 \beta T}}
$$

and

$$
u_{i}=\frac{g T}{1-e^{-2 \beta T}}-\frac{g}{2 \beta}-2 v_{o}
$$

Eqs.(22) and (23) can be solved graphically for $\beta$ and $u_{i}$ as functions of the wave period and amplitude. The velocity $u_{i}$ and the damping parameter of the converter can then be used to estimate the extractable power. The damping parameter can be derived as follows.

\section{Deriving $\beta$}

The parameter $\beta$, as defined in Eq.(17), encompasses the total energy leaving the system during each cycle and has two contributions: one term due to friction and irreversible losses $\left(\beta_{f}\right)$, and another term associated with the converter $\left(\beta_{c}\right)$. Eq.(17) can thus be rewritten

$$
\beta=\beta_{c}\left[1+\frac{\beta_{f}}{\beta_{c}}\right]
$$

If $\beta_{c} \gg \beta_{f}$, then $\beta \approx \beta_{c}$ (see Appendix). In terms of the slingshot effect, the most convenient converter possible is clearly one that is driven by a linear inductance, as proposed by [17]. This scenario involves a permanent magnet moving inside a coil. It could be implemented in the present case by placing a coil in the tower and attaching a permanent magnet to the puck. The electrical power $P$ produced by such a converter is then given by [17]

$$
P=\left[\frac{N^{2} B^{2} l^{2}}{R}\right] u^{2},
$$

where $N$ is the number of turns of the wire loop, $B$ is the magnetic induction, $l$ is the length of the wire within the magnetic field, $R$ is the load resistance in the wire loop, and $u$ is the puck velocity.

Current flow results in a force acting on the loop and on the magnet, in a direction opposite to the puck velocity:

$$
F=\frac{P}{u} .
$$

We then have

$$
F=b u
$$

with

$$
b=\frac{N^{2} B^{2} l^{2}}{R} .
$$

Using Eq.(16), one obtains

$$
\beta_{c}=\frac{N^{2} B^{2} l^{2}}{2 m_{p} R}
$$

or, with our approximation $\beta \approx \beta_{c}$ (see Appendix),

$$
\beta=\frac{N^{2} B^{2} l^{2}}{2 m_{p} R}
$$

Likewise, the power generated by the electromagnetic inductor is given by Eq.(25), which can be rewritten as

$$
P=2 m_{p} \beta u^{2} .
$$

The time-averaged power derived from the linear inductance device over one cycle is

$$
\begin{gathered}
\bar{P}=\frac{1}{T} \int_{0}^{T} P d t \\
=\frac{2 m_{p} \beta}{T} \int_{0}^{T} u^{2} d t .
\end{gathered}
$$

Inserting Eq.(20), we have

$\bar{P}=\frac{2 m_{p} \beta}{T} \int_{0}^{T}\left[-\frac{g}{2 \beta}\left[1-e^{-2 \beta t}\right]+\left(u_{i}+2 v_{o}\right) e^{-2 \beta t}\right]^{2} d t$ 
which after integration yields

$$
\bar{P}=m_{p}[X-Y]
$$

with

$$
X=\frac{g^{2}}{2 \beta}+\frac{1}{2 T}\left[\frac{g}{2 \beta}+u_{i}+2 v_{o}\right]^{2}
$$

and

$$
Y=\frac{1}{2 T}\left[\frac{g}{\beta}-\left(\frac{g}{2 \beta}+u_{i}+2 v_{o}\right) e^{-2 \beta T}\right]^{2},
$$

where, for a given ocean wave, the period $T$ and the velocity amplitude $v_{o}$ are related via $v_{o}=\frac{2 \pi H}{T}$, with $H$ being the wave amplitude. Parameters $\beta$ and $u_{i}$ are determined by solving Eqs.(22) and (23) simultaneously.

\section{- Discussion III}

To estimate the power extractable from a slingshot resonant tower predicted by the above analysis, we assume typical parameter values, i.e., a wave with amplitude $H=0.6 \mathrm{~m}$ and a puck mass of $m_{p}=2 \mathrm{~kg}$. The predicted power and puck velocity $u_{i}$ at the moment of collision are plotted in Fig. 4 as functions of the wave period.

These results suggest that the slingshot resonance can yield a power in excess of $20 \mathrm{~kW}$ for ocean waves with a period of $10 \mathrm{~s}$ (the swells). However, this is not the case. The slingshot effect requires that the puck momentum be much less than the wave momentum. It is therefore only approximately valid for a puck of finite mass and velocity. The slingshot analysis cannot therefore apply with equal accuracy to all tower configurations.

The maximum height attained by the puck (and hence the required tower height) is plotted in Fig. 5 as a function of the wave period, from Eq.(21). A slingshot tower is thus practically feasible for ocean waves of period less than $\approx 4$ s. According to Fig. 4, such a tower, with a height of order $20 \mathrm{~m}$, would generate a power of order 20 $\mathrm{kW}$.

\section{SUMMARY OF RESULTS AND CONCLUSIONS}

We have outlined a novel concept for converting wave energy based on the so-called slingshot effect. Energy extraction proceeds from repeated elastic collisions of a puck with a pusher plate that is driven by the wave motion, in a way that significantly amplifies the vertical kinetic energy of the puck. By considering a simplified geometrical model, we demonstrated that, despite the stochastic nature of the collisions (whereby collisions occur at different points of the wave cycle), head-on collision occur more frequently on average, resulting in a net gain of energy. The most promising application of this oceanic slingshot effect is the possibility of matching, through appropriate design, the travel time of the puck between collisions with the period of the ocean wave, such that only head-on collisions occur, leading to an optimal magnification of the puck kinetic energy. This kind of resonance is specially well suited to high-frequency ocean waves. In contrast, existing wave converters are optimised for long-period waves (swells).

\section{APPENDIX}

Previous analyses made the simplifying assumption that frictional damping is negligible compared with the effective damping associated with the linear inductance converter, i.e., $\beta_{c} \gg \beta_{f}$. However, this approximation should be assessed explicitly.

The frictional damping factor is mostly due to atmospheric drag. The atmospheric drag force $F_{d}$ acting on a cylindrical cavity of diameter $D_{c}$ with a cylindrical puck of approximately the same diameter (see Fig. 3) is given by

$$
F_{d} \approx \frac{c_{d} \rho \pi D_{c}^{2} u^{2}}{8}
$$

where $c_{d}$ is the drag coefficient, $\rho$ is the atmospheric density, $D_{c}$ the cavity diameter (approximately equal than the puck diameter), and $u$ is the relative velocity of the puck and the atmosphere. Compared with Eq.(15), the damping factor yields

$$
\beta_{f}=\frac{c_{d} \rho \pi D_{c}^{2} u}{16 m_{p}}
$$

with a drag coefficient $c_{d} \sim 0.4$. For $\rho=1 \mathrm{~kg} / \mathrm{m}^{3}$ and a cavity $D_{c}=0.2 \mathrm{~m}$ with a somewhat heavy puck $m_{p}=100 \mathrm{~kg}$, we have $\beta_{f} \approx 3 \times 10^{-5} u$. The velocity cannot exceed $u \sim 10 \mathrm{~m} / \mathrm{s}$, as the puck momentum would otherwise become significant in comparison with the wave momentum, which would invalidate the slingshot approximation for the elastic collision. Therefore, with $\beta_{c} \approx 0.2$, frictional damping can be neglected in comparison with the damping resulting from the linear inductance.

\section{NOMENCLATURE}

$b=$ damping coefficient

$B=$ magnetic field

$c_{d}=$ drag coefficient

$C_{1}=$ constant

$C_{2}=$ constant

$D_{c}=$ slingshot cavity diameter

$E=$ energy

$F=$ forces 


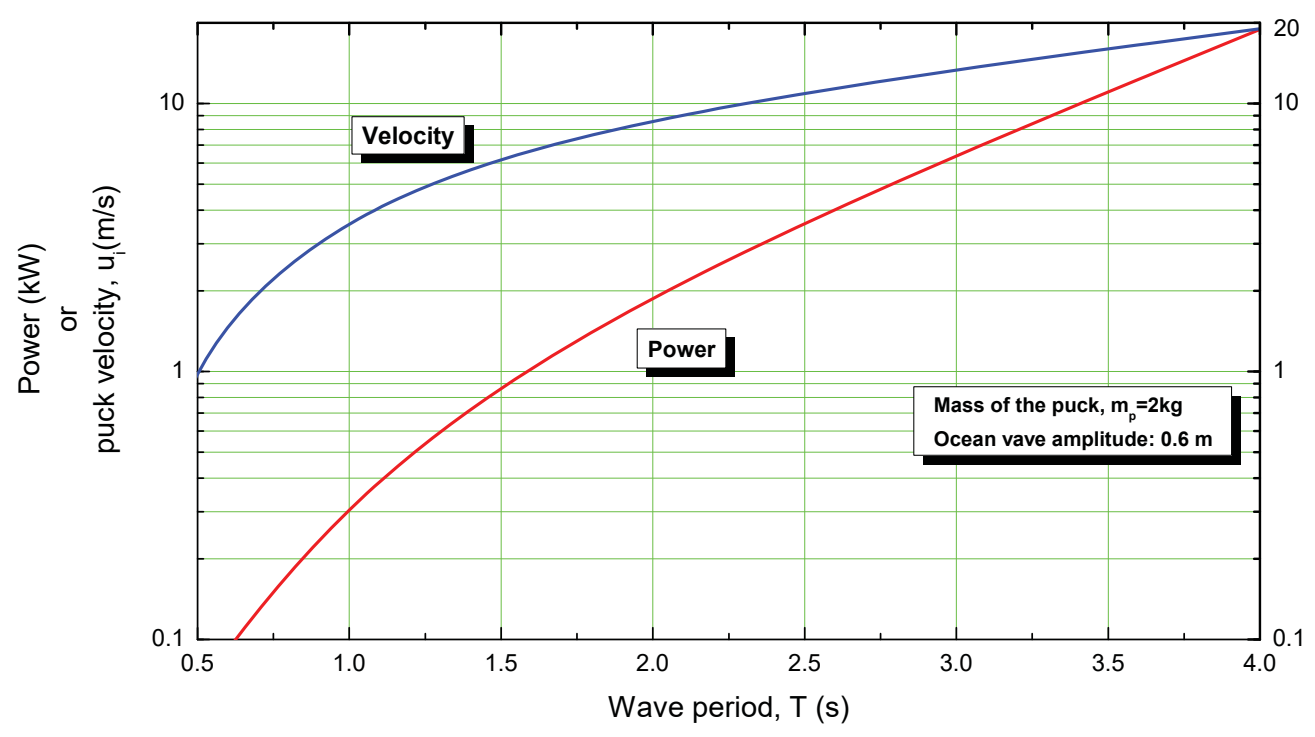

FIG. 4: Energy extractable from a slingshot resonant cavity, as a function of the wave period.

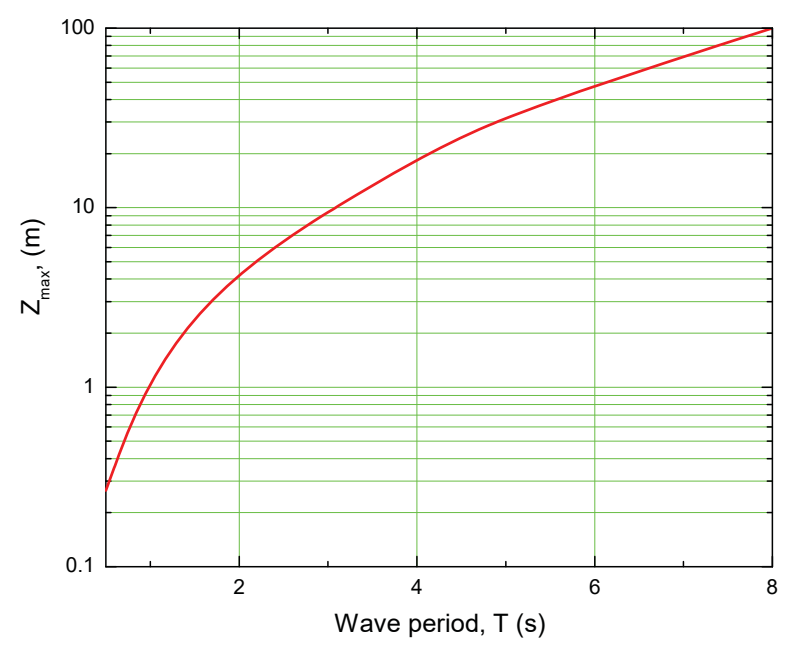

FIG. 5: Height of a slingshot resonant tower as a function of the wave period.

$g=$ gravity

$h=$ cavity length

$H=$ wave amplitude

$l=$ wire length

$m_{p}=$ puck mass

$N=$ number of turns in the wire loop

$P=$ power

$R=$ load resistance

$t=$ time

$T=$ wave period

$u=$ puck velocity $v=$ ocean wave velocity

$X=$ parameter, Eq.(34)

$Y=$ parameter, Eq.(35)

$z=$ vertical length co-ordinate

\section{Greek symbols}

$\beta=$ total damping parameter

$\beta_{f}=$ damping parameter due to friction

$\beta_{c}=$ damping parameter due to the converter

$\delta=$ phase difference between the puck and the wave

$\rho=$ atmospheric density

$\omega=$ wave frequency

$\Gamma=$ net gain in the resonant mode

\section{subscripts}

$c=$ converter

$d=$ downwards

$f=$ friction

$r=$ resonant

$s=$ stochastic

$u=$ upwards

\section{ACKNOWLEDGEMENTS}

This research was supported by the Spanish Ministry of Economy and Competitiveness under the fellowship grant Ramon y Cajal: RYC-2013-13459.

\section{References}


[1] Ross, D. 1995. Power from the Waves. Oxford University Press, Oxford, UK

[2] Drew B., Plumer A.R., Sahinkaya M.N., 2009. A Review of Wave Energy Converter Technology. Proc. IMechE Vol. 223, Part A: J. Power and Energy.

[3] Rusu E., Onea F. 2018. A review of the technologies for wave energy extraction. Clean Energy, 2,(1). p.p. 10-19

[4] Benbouzid H.T; Benbouzid M. 2015. An Up-to-Date Technologies Review and Evaluation of Wave Energy Converters. International Review Of Electrical Engineering-Iree, 10, (1), pp. 52-61

[5] Budal K. Falnes J. 1975. A resonant point absorber of ocean-wave power. Nature. 256, pp. 478-479

[6] Falnes J. 2002. Ocean Waves and Oscillating Systems. Cambridge University Press, Cambridge, England, pp. $1-275$.

[7] Miles J.N. 1971. Resonant response of harbours: an equivalent circuit analysis. Journal of Fluid Mechanics, 46, 241-265.

[8] Engströma J, Kurupath V; Isberg J., Leijon M. 2011. A resonant two body system for a point absorbing wave energy converter with direct-driven linear generator. Journal of Applied Physics 110, 124904

[9] McCormick M.E. 2007. Ocean Wave Energy Conversion. Dover Publications, Inc. Mineola, New York.
[10] Masuda Y. 1971. Wave Activated generator . Proceeding, International Collowium on the Exposition of teh oceasn, Bordeaux, France, March.

[11] Budal K., Falnes J. 1980. Power from Sea Waves. B. M. Count, Academic Press, London/New York

[12] Falnes J. 2002. Int. J. Offshore Polar Eng. 12(2), 147 (2002).

[13] Babarit A., Duclos G., Clement A.H. 2004. Comparison of latching control strategies for a heaving wave energy device in random sea. Appl. Ocean. Res. 26, 227.

[14] Schoen M.P., Hals J., Moan T. 2011. Wave Prediction and Robust Control of Heaving Wave Energy Devices for Irregular Waves IEEE Trans. Energy Convers. 99, 13.

[15] Engström J; Eriksson M., Isberg J; Leijon M. 2009. Wave energy converter with enhanced amplitude response at frequencies coinciding with Swedish west coast sea states by use of a supplementary submerged body. J. Appl. Phys. 106, 064512.

[16] Candido J.J; Justino P.A.P.S. 2010. Modelling, control and Pontryagin Maximum Principle for a two-body wave energy device. Renewable Energy 36, 1545.

[17] Omholt T. 1978. A wave Activated Electric Generator. Proceedings, Ocean 78, marine Technology Conference, Washington, D.C, pp. 585-589. 